\title{
Formulation design, preparation, and in vitro and in vivo characterizations of $\beta$-Elemene- loaded nanostructured lipid carriers
}

This article was published in the following Dove Press journal:

International Journal of Nanomedicine

19 July 2013

Number of times this article has been viewed

\author{
Feng Shi' \\ Gang Yang' \\ Juan Ren ${ }^{2}$ \\ Teng Guo' \\ Yan Du' \\ Nianping Feng' \\ 'Department of Pharmaceutics, \\ School of Pharmacy, Shanghai \\ University of Traditional Chinese \\ Medicine, Shanghai, People's Republic \\ of China; ${ }^{2}$ Department of Traditional \\ Chinese Medicine, Changhai Hospital, \\ Shanghai, People's Republic of China
}

Correspondence: Nianping Feng Department of Pharmaceutics, School of Pharmacy, Shanghai University of Traditional Chinese Medicine, 1200 Cailun Road, Zhangjiang Hi-Tech Park, Pudong New District, Shanghai 20I203, People's Republic of China Tel +8621 51322198 Email npfeng@hotmail.com

\begin{abstract}
In the present study, nanostructured lipid carriers (NLCs) were prepared and optimized for the intravenous delivery of $\beta$-Elemene $(\beta-\mathrm{E})$. Aqueous dispersions of NLCs were successfully prepared by high-pressure homogenization method using glycerol monostearate as the solid lipid and a mixture of Maisine 35-1 and Labrafil M1944 CS as the liquid lipid. The results revealed that the morphology of the NLCs was spheroidal. The particle size, zeta potential, and entrapment efficiency (EE) for the optimized formulation were observed as $138.9 \mathrm{~nm},-20.2 \mathrm{mV}$, and $82.11 \%$, respectively. X-ray diffraction analysis revealed the formation of less ordered structures in the inner core of the NLC particles. Moreover, the $\beta$-E-loaded NLCs were also less irritating and less toxic compared to Elemene injection. In addition, $\beta$-E-NLCs showed a significantly higher bioavailability and anti-tumor efficacy than Elemene injection. Taken together, our data indicate that the $\beta$-E-NLCs described in this study are well-suited for the intravenous delivery of $\beta$-E.
\end{abstract}

Keywords: nanostructured lipid carriers, high-pressure homogenization, $\beta$-Elemene, venous irritation, anti-tumor efficacy

\section{Introduction}

Over the past several years, considerable attention has been focused on the development of nanosized drug delivery systems for Chinese herbal medicines. Novel nanoformulations of Chinese herbal medicines, such as nanoemulsions, liposomes, and polymeric nanoparticles, have been reported to demonstrate remarkable advantages over conventional formulations. ${ }^{1}$ In addition, many studies have shown that nanosized drug delivery systems can enhance the efficacy and reduce toxicity of drugs. ${ }^{2,3}$ Importantly, nanotechnology has opened up new research avenues for the development and modernization of Chinese medicine. Currently, many specialists in the People's Republic of China regard the application of nanotechnology as an effective way to internationalize Chinese medicine. ${ }^{4}$

Recently, lipid nanoparticles consisting of biocompatible lipids have become attractive targets for the delivery of poorly soluble drugs. ${ }^{5}$ Solid lipid nanoparticles (SLNs) and nanostructured lipid carriers (NLCs) are two general types of lipid nanoparticles. SLNs are prepared using solid lipids, which are biocompatible and biodegradable, have several advantages, including greater stability, negligible toxicity, and biodegradability. ${ }^{6} \mathrm{NLCs}$, which consist of a mixture of solid and liquid lipids, have evolved as second-generation lipid nanoparticles to overcome the disadvantages of SLNs, including larger particle sizes, limited drug-loading capacity, and risk of gelation. ${ }^{7,8}$ 
$\beta$-Elemene, $(5 S, 7 R, 10 S)-(-)-(1-m e t h y l-1-v i n y l-2,4-$ diisopropenyl-cyclohexane; $\beta-E)$, is a natural, volatile, anti-neoplastic agent extracted from the traditional Chinese medicinal herb Rhizoma Curcumae. ${ }^{9} \beta$-E exerts anti-cancer effects in a variety of tumors, such as liver cancer, brain cancer, lung cancer, and others. ${ }^{10-12}$ Previous studies have demonstrated that the anti-cancer activity of $\beta$-E is mainly owing to its inhibition of tumor cell growth and induction of tumor cell death in vitro and in vivo. ${ }^{13}$ In the People's Republic of China, $\beta$-E has been used in clinical settings (via injection) to treat patients with malignant tumors, including lung cancer and liver cancer, ${ }^{14}$ and it has also been studied in clinical trials conducted in the US. ${ }^{15}$

However, despite its beneficial pharmacological effects, $\beta$-E suffers from poor water solubility and limited bioavailability. ${ }^{15}$ In addition, $\beta$-E injection can cause serious venous irritation and phlebitis when used clinically. ${ }^{16}$ Taken together, these factors limit the clinical application of $\beta$-E, and therefore, a suitable formulation of the drug is required to overcome these drawbacks. In this study, we aimed to identify an optimal formulation of $\beta$-E for enhancing its anti-cancer effects and reducing venous irritation. $\beta$-E-loaded NLCs ( $\beta$-E-NLCs) were prepared, and physical properties such as particle size, zeta potential, and other properties were evaluated. In addition, its pharmacokinetics, anti-cancer effects, and venous irritation were investigated to estimate the potential safety and efficacy of $\beta$-E-NLCs for clinical use.

\section{Materials and methods Materials}

$\beta$-E was purchased from Yueqing Institute of zedoary turmeric oil (Zhejiang, People's Republic of China). The Elemene injection was purchased from Dalian Jingang Medicine Company (Dalian, People's Republic of China). Glycerol monostearate (GMS) and polysorbate 80 (Tween $80^{\circledR}$ ) were supplied by the China National Pharmaceutical Group Corporation (Shanghai, People's Republic of China). Glyceryl monolinoleate (Maisine 35-1 ${ }^{\circledR}$ ) and oleoyl macrogolglycerides (Labrafil M1944 CS ${ }^{\circledR}$ ) were provided by Gattefosse France (Gennevilliers, France). Soybean lecithin (Lipoid S 100) was donated by Lipoid GmbH (Ludwigshafen, Germany). All of the organic solvents were of analytical reagent grade.

\section{Animals}

Kunming strain mice (20 $\pm 2 \mathrm{~g}$ ), New Zealand White rabbits $(3.0 \pm 0.3 \mathrm{~kg})$, and Wistar rats $(200 \pm 20 \mathrm{~g})$ were purchased from the Laboratory Animal Center of the Shanghai University of Traditional Chinese Medicine (Shanghai, People's Republic of China). All of the animals were acclimatized at a temperature of $25^{\circ} \mathrm{C} \pm 2{ }^{\circ} \mathrm{C}$ and a relative humidity of $70 \% \pm 5 \%$ under natural conditions for 1 week with food and water ad libitum. All of the experimental procedures were performed according to the guidelines of the Animal Ethical Committee at Shanghai University of Traditional Chinese Medicine.

\section{Preparation of $\beta$-E-NLCs}

$\beta$-E-NLCs were prepared by hot melting high-pressure homogenization method. ${ }^{17}$ Briefly, the lipids consisted of a mixture of solid (GMS) and liquid lipids (Maisine 35-1 and Labrafil M1944 CS), which were heated at $65^{\circ} \mathrm{C}$ until melted. Next, the drug was added as the organic phase. The aqueous phase, which contained Tween 80 and soybean lecithin in double-distilled water, was prepared at the same temperature. Next, the aqueous phase was dispersed into the organic phase at $65^{\circ} \mathrm{C}$ under high-speed mixing using an Ultra Turrax T25 (IKA, Staufen, Germany). The resulting pre-emulsion was then immediately passed through a high-pressure homogenizer (NS1001 L, GEA, Parma, Italy). The $\beta$-E-NLCs were obtained by solidification in an ice bath. This production technology and formulation have been optimized via a single factor and orthogonal experiment, respectively.

\section{Entrapment efficiency (EE) and drug loading (DL)}

The free drug was separated from the drug entrapped in the NLCs using a previously described ultrafiltration method. ${ }^{18}$ Briefly, the desired amounts of $\beta$-E-NLCs were diluted with $0.5 \%$ (by weight) phosphate buffer solution with Tween 80 to dissolve the free drug. ${ }^{19}$ The solution was centrifuged for 15 minutes at $8000 \mathrm{rpm}$ using centrifugal filter tubes (10 kDa; Pall Corporation, Port Washington, NY, USA). The total drug and separated drug contents were measured using gas chromatography spectrometry (GC) under the following conditions: Agilent 7890A GC system coupled with a flame ionization detector and an Agilent 19091-413 HP-5 capillary column $(30 \mathrm{~m} \times 0.32 \mathrm{~mm} \times 0.25 \mu \mathrm{m})$, with the temperature set to increase from $60^{\circ} \mathrm{C}$ to $200^{\circ} \mathrm{C}$ at a rate of $10^{\circ} \mathrm{C}$ /minute. Nitrogen was used as the carrier, and the samples were determined in a splitless model. The EE and DL were mathematically calculated using equations (1) and (2) below:

$$
\begin{aligned}
& \mathrm{EE} \%=\frac{W_{\text {total }}-W_{\text {free }}}{W_{\text {total }}} \times 100 \\
& \mathrm{DL} \%=\frac{W_{\text {total }}-W_{\text {free }}}{W_{\text {lipids }}} \times 100
\end{aligned}
$$


where $W_{\text {total }}, W_{\text {free }}$, and $W_{\text {lipids }}$ are the total amount of $\beta$-E present in the $\beta$-E-NLCs dispersion, the amount of untrapped $\beta$-E, and the amount of lipids added to the $\beta$-E-NLCs dispersion, respectively.

\section{Physicochemical characterization of $\beta$-E-NLCs}

Particle size and zeta potential

The mean diameter, polydispersity index (PDI), and zeta potential of the NLCs were measured using a Nano ZS90 Zetasizer (Malvern Instruments Ltd, Worcestershire, UK). The zeta potential was calculated using the Smoluchowski equation. The samples were appropriately diluted with distilled water and the particle size was evaluated using volume distribution.

\section{Morphology}

The morphology of the $\beta$-E-NLCs was examined using a transmission scanning microscope (TEM; JEM-1230, Tokyo, Japan). Initially, the samples were diluted with distilled water, followed by placement on a film-coated copper grid, where the samples were then stained with a drop of $2 \%$ phosphotungstic acid and allowed to dry before examination under the TEM.

\section{$X$-ray diffraction (XRD)}

XRD analysis of the $\beta$-E-NLCs was performed using an X-ray diffractometer (Rigaku Corporation, Tokyo, Japan) to assess the crystalline structures. The diffraction pattern was measured at $2 \theta$ values of $2^{\circ}$ to $50^{\circ}$ at a scanning rate of $5 \%$ minute using a $\mathrm{Cu}-\mathrm{K} \alpha$ radiation source. Prior to examination, the $\beta$-E-NLCs were frozen using mannitol (8\%) as the cryoprotecting agent.

\section{Venous irritation}

New Zealand White rabbits were used to evaluate the venous irritation of the $\beta$-E-NLCs. ${ }^{20}$ The rabbits were divided into three groups (Groups A, B, and C) with each group containing three rabbits. Groups $\mathrm{A}$ and $\mathrm{B}$ were given an injection of Elemene (used in the clinic) or the $\beta$-E-NLC suspension, respectively, into the ear vein. The dosage used was set at $10 \mathrm{mg} / \mathrm{kg}$ ( $\beta$-E equivalent dose) on the basis of the dose used in humans. Group $\mathrm{C}$ was given an equivalent volume of saline solution in the same manner as Groups A and B. The administration rate was set to $1 \mathrm{~mL} /$ minute for these three groups and was repeated after 5 days. For the injection, visual examination of the injection site was performed to investigate the paradoxical reaction. The rabbits were then sacrificed 12 hours after the last injection, followed by fixation of the vascular tissues with a $10 \%$ buffer-formalin solution for histopathological examination.

\section{Pharmacokinetics}

The bioavailability of the Elemene injection was compared to that of the $\beta$-E-NLC suspension. Male Wistar rats were used in these experiments and were divided into two groups $(n=6)$. An Elemene injection or $\beta$-E-NLC suspension was given intravenously to the rats at a dose of $40 \mathrm{mg} / \mathrm{kg}$ (based on the dose used in humans). After the injection, approximately $0.5 \mathrm{~mL}$ of blood was collected from the ocular vein into a heparinized tube at 5, 10, 20, 30, 40, 50, 60, and 80 minutes. The collected blood samples were centrifuged at $5000 \mathrm{rpm}$ for 10 minutes. The obtained plasma samples were then stored at $-70^{\circ} \mathrm{C}$ prior to $\mathrm{GC}$ analysis under the same conditions previously described in the EE and DL section above.

Pharmacokinetic parameters such as clearance (CL), and area under the concentration-time curve (AUC) were calculated using the pharmacokinetic software, 3P97 (Mathematics Pharmacological Committee of the Chinese Academy of Pharmacology, Shanghai, People's Republic of China).

\section{In vivo anti-tumor efficacy}

Evaluation of in vivo anti-tumor efficacy was performed as previously described. ${ }^{21}$ Briefly, $\mathrm{H}_{22}$ cells (obtained from Chinese Academy of Sciences, Shanghai, People's Republic of China) were injected into the abdominal cavity of mice for 6-8 days before they were collected and resuspended in phosphate buffered saline (PBS). Kunming mice were divided into four groups (10 mice/group), followed by subcutaneous inoculation with $\mathrm{H}_{22}$ tumor cells into the right axilla at a concentration of $0.2 \mathrm{~mL}\left(2 \times 10^{6} / \mathrm{mL}\right)$. After inoculation, Groups 1, 2, and 3 were continuously injected with either Elemene injection ( $\beta$-E equivalent dose of $60 \mathrm{mg} / \mathrm{mL}$ ), $\beta$-E-NLC suspension ( $\beta$-E equivalent dose of $60 \mathrm{mg} / \mathrm{mL}$ ) or saline (control drug) via the tail vein. The mice were then administered with Fluorouracil (5-FU), as a reference drug which is normally used in the clinic for treatment of liver cancer, once every 2 days at a dose of $25 \mathrm{mg} / \mathrm{kg}$ via the tail vein. Ten days later, all of the animals were sacrificed by cervical dislocation, and the tumors, spleens, and thymi were isolated and weighed. The tumor growth inhibition rate, and the spleen and thymus indices were calculated using equations (3) and (4):

$$
\text { Tumor inhibitory rate }(\%)=\frac{\mathrm{C}-\mathrm{T}}{\mathrm{C}} \times 100
$$




$$
\text { Spleen/Thymus index }=\frac{W_{S / T}}{W_{M}}
$$

where $\mathrm{C}$ and $\mathrm{T}$ are the tumor weight average of the control and treated groups; $W_{S / T}$ is the weight of the spleen or thymus; and $W_{M}$ represents the weight of the corresponding mouse.

\section{Statistical analysis}

Data are expressed as the mean \pm standard deviation. Statistical significance was determined using Student's $t$-test with $P<0.05$ indicating significance.

\section{Results and discussion Technology and formulation optimization of the $\beta$-E-NLCs}

The preparation technology was optimized using a single factor experiment, using the production parameters including the stirring power (watt), stirring time (minute), homogenization pressure (bar), and homogenization cycle as the factors, and the particle size and PDI as the indices. All of the other factors were fixed when one factor was being investigated; these results are shown in Figure 1. As shown in Figure 1B, the prepared nanoparticles were dependent on
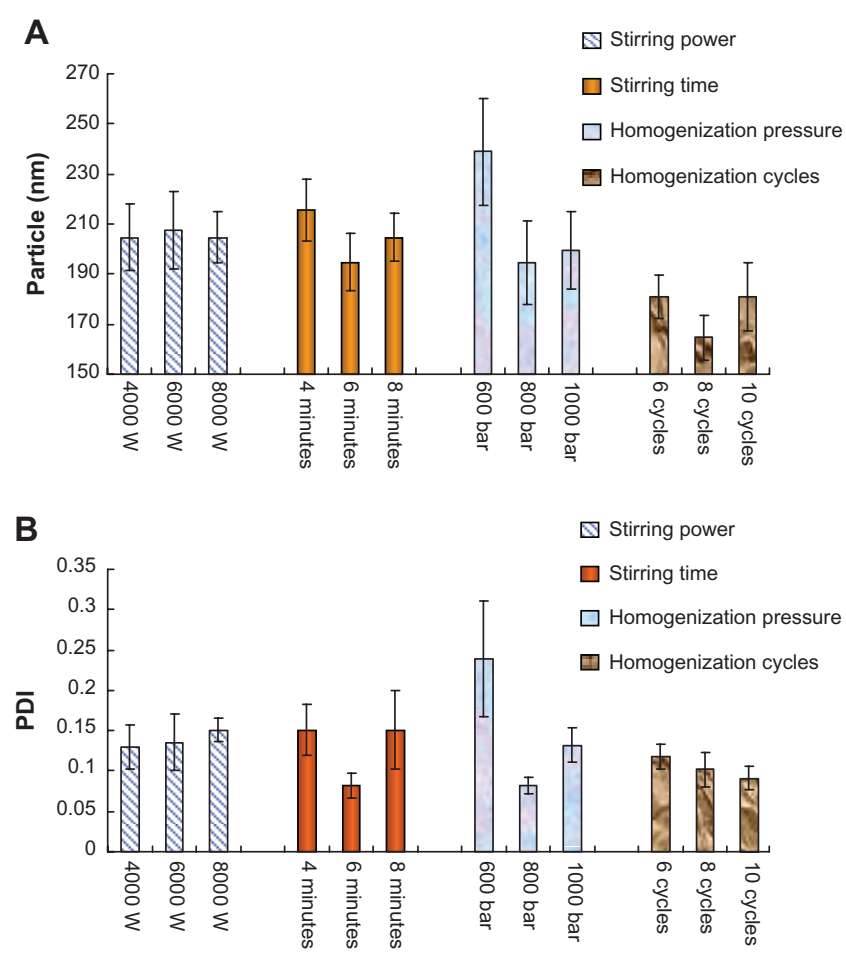

Figure I Optimization for the technical preparation of the $\beta$-E-NLCs. Stirring power, stirring time, homogenization pressure, and homogenization cycle are used as the factors, and the particle size (A) and PDI (B) are used as the index.

Abbreviations: $\beta$-E-NLCs, $\beta$-Elemene-nanostructured lipid carriers; PDI, polydispersity index. various factors, all of which received an ideal PDI value $(<0.25)$. Stirring power had no apparent effect on particle size and PDI. Moreover, as homogenization pressure and homogenization cycle increased, the particle size was subsequently reduced followed by a slight increase. It has been previously reported that the temperature of the preparation system decreases with an increase in the homogenization pressure and homogenization cycle, resulting in an increase in the kinetic energy. ${ }^{22}$ Thus, the particles are likely to reassemble when prepared. On the basis of the single factor experiment, the production parameters were set at a stirring power of 4000 watts, a stirring time of 6 minutes, a homogenization pressure of 800 bars, and a homogenization cycle of 8 .

Formulation optimization of $\beta$-E-NLCs was performed using orthogonal experiments. Prior to the orthogonal experiments, we performed preliminary studies to analyze different types of lipids and surfactants, and the weight of the drug required for adding to the preparation. GMS, a mixture of Maisine 35-1 and Labrafil M1944 CS (at a ratio of 1:1), and a mixture of Tween 80 and soybean lecithin (at a ratio of 1:1) were selected as the solid lipid, liquid lipid, and surfactant, respectively. On the basis of the preliminary experiments, four influential factors including the concentration of the total lipids (L, \%), surfactants (S, \%), weight ratio of the solid lipid to liquid lipid (S/L, weight/weight $[\mathrm{w}: \mathrm{w}]$ ), and the weight ratio of the drug to the total lipids $(\mathrm{D} / \mathrm{L}, \mathrm{w} / \mathrm{w})$ were optimized using $\mathrm{L}_{9}\left(3^{4}\right)$ orthogonal experiments by taking the EE as the index.

Four factors and their three levels are listed in Table 1, with the results being shown in Table 2. The Rj (range) value of $\mathrm{L}$ was the highest among the four factors, indicating its effect on EE. EE was also enhanced by increases in lipid concentration. The optimal formulation was as follows (which was dependent on $\mathrm{Rj}$ and $\mathrm{K}$ [mean value]): the concentration of lipids and surfactants, $\mathrm{S} / \mathrm{L}$ ratio, and $\mathrm{D} / \mathrm{L}$ ratio were $6 \%, 5 \%, 2: 3$, and 1:20, respectively. Since the $\mathrm{D} / \mathrm{L}$ ratio did not appreciably affect EE, it was established at 1:10 in order to increase $\mathrm{D} / \mathrm{L}$ and reduce the administration dosage. The optimized formulation was prepared in triplicate.

Table I The four factors and their three levels set for the orthogonal experiment

\begin{tabular}{lllll}
\hline Factors/levels & L (\%) & S (\%) & S/L (w/w) & D/L (w/w) \\
\hline $\mathrm{I}$ & 2 & 4 & $3: 2$ & $\mathrm{I}: 10$ \\
2 & 4 & 5 & $\mathrm{I}: \mathrm{I}$ & $\mathrm{I}: 15$ \\
3 & 6 & 6 & $2: 3$ & $\mathrm{I}: 20$ \\
\hline
\end{tabular}

Abbreviations: L, lipid; S, surfactant; w, weight; D, drug. 
Table 2 Results of the $\mathrm{L}_{q}\left(3^{4}\right)$ orthogonal experiment

\begin{tabular}{|c|c|c|c|c|c|}
\hline Factors & L (\%) & S (\%) & $S / L(w / w)$ & $D / L(w / w)$ & EE (\%) \\
\hline I & 2 & 4 & $3: 2$ & $1: 10$ & 61.60 \\
\hline 2 & 2 & 5 & $I: I$ & $1: 15$ & 72.40 \\
\hline 3 & 2 & 6 & $2: 3$ & $\mathrm{I}: 20$ & 65.71 \\
\hline 4 & 4 & 4 & I:I & $1: 20$ & 70.20 \\
\hline 5 & 4 & 5 & $2: 3$ & $1: 10$ & 76.30 \\
\hline 6 & 4 & 6 & $3: 2$ & $1: 15$ & 68.51 \\
\hline 7 & 6 & 4 & $2: 3$ & $1: 15$ & 78.30 \\
\hline 8 & 6 & 5 & $3: 2$ & $\mathrm{I}: 20$ & 84.21 \\
\hline 9 & 6 & 6 & $\mathrm{I}: \mathrm{I}$ & $1: 10$ & 76.50 \\
\hline$K_{1}$ & 66.57 & 70.03 & 71.43 & 71.47 & \\
\hline $\mathrm{K}_{2}$ & 71.67 & 77.63 & 73.03 & 73.07 & \\
\hline $\mathrm{K}_{3}$ & 79.67 & 70.23 & 73.43 & 73.37 & \\
\hline $\mathrm{Rj}$ & $13 . \mid$ & 7.6 & 2 & 1.9 & \\
\hline
\end{tabular}

Abbreviations: $K$, mean value; $R j$, range; $D$, drug; $E E$, encapsulation efficiency L, lipid; S, surfactant; w, weight.

\section{EE and DL}

The average EE and DL of the freshly prepared $\beta$-E-NLCs were $82.11 \% \pm 1.84 \%$ and $8.45 \% \pm 0.57 \%$, respectively. $\mathrm{EE}$ and DL are important factors used to judge the suitability of the lipid nanoparticles. ${ }^{23}$ Several methods have been used to investigate $\mathrm{EE}$, such as mini-column centrifugation, ${ }^{24}$ dialysis, ${ }^{25}$ and ultrafiltration, which was used in the present study. Compared to other methods, the ultrafiltration method is convenient and can separate the free drug without destroying the carriers. ${ }^{26}$ In our preliminary study, we diluted the $\beta$-E-NLCs in distilled water prior to ultrafiltration. However, because of its poor water solubility, the separated $\beta$-E was not detected using GC. Thus, $0.5 \%$ (by weight) Tween 80 phosphate buffer solution was used to dilute the $\beta$-E-NLCs in order to solubilize the free drug.

\section{Nanoparticle characterization}

The mean diameter of the freshly prepared $\beta$-E-NLCs was $138.9 \pm 4.7 \mathrm{~nm}$ with a particle diameter size that ranged from 60 to $400 \mathrm{~nm}$, and the PDI value was 0.085 , indicating a relatively narrow size distribution. The zeta potential of the $\beta$-E-NLCs, which may be regarded as a prediction of storage stability of the solid nanoparticles, ${ }^{27}$ was $-20.2 \pm 1.1 \mathrm{mV}$, indicating a negatively charged surface on the nanoparticles.

The results of TEM imaging of the $\beta$-E-NLCs are shown in Figure 2, revealing that the $\beta$-E-NLCs were round in shape with a uniform size.

In addition to particle size and zeta potential, characterization of the crystallinity of the NLCs is also important. In the present study, lyophilized $\beta$-E-NLCs were obtained by freezedrying for XRD determination. The X-ray patterns of the GMS, cryoprotecting agent (mannitol), and $\beta$-E-NLCs are shown in

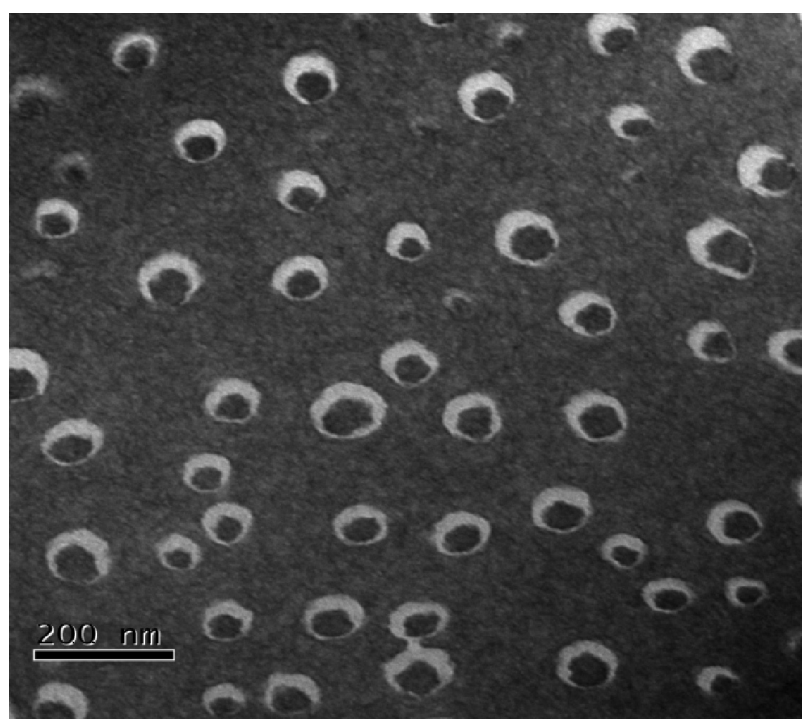

Figure 2 TEM images of the $\beta$-E-NLCs.

Abbreviations: $\beta$-E-NLCs, $\beta$-Elemene-nanostructured lipid carriers; TEM, transmission scanning microscope.

Figure 3. The diffraction pattern of GMS was significantly different from that of the $\beta$-E-NLCs. GMS showed diffraction peaks at $2 \theta$ values of $5.5^{\circ}, 7.4^{\circ}, 19.5^{\circ}, 20.6^{\circ}$, and $23.4^{\circ}$ (Figure 3A). However, five characteristic peaks of GMS disappeared, as shown in Figure 3C, and peak intensity decreased significantly compared to those shown in Figure 3A. Moreover, the blunt peak depicted in Figure $3 \mathrm{C}$ at the $2 \theta$ value of $23.5^{\circ}$ may be a result of the cryoprotecting agent (Figure 3B).$^{28}$ The results of the XRD determination confirmed that GMS exhibited a disordered crystalline structure in the NLCs. Of note, XRD analysis of the pure drug or the liquid lipids was not performed because of their liquid state.

\section{Venous irritation}

Venous irritation is one of the most unpleasant toxicities experienced by cancer patients. ${ }^{29}$ Essential oils, such as

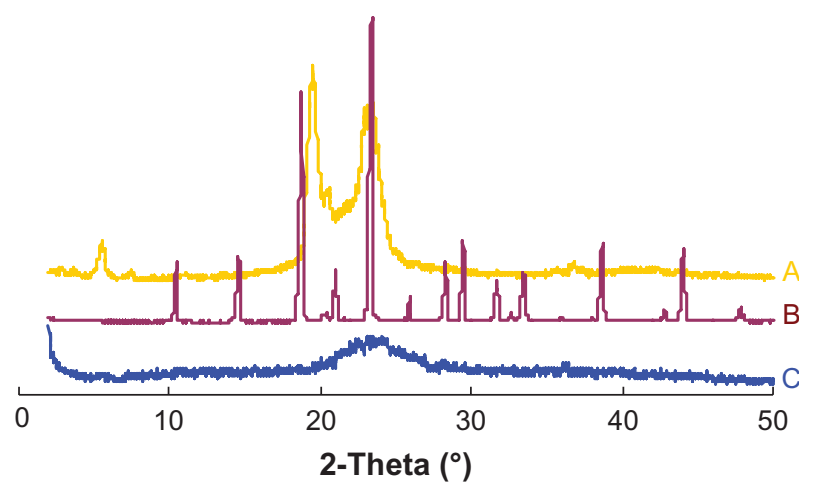

Figure 3 X-ray patterns of GMS (A), mannitol (B), and $\beta$-E-NLCs (C). Abbreviations: $\beta$-E-NLCs, $\beta$-Elemene-nanostructured lipid carriers; GMS, glyceryl monostearate. 
$\beta$-E, are well known to cause venous irritation including phlebitis and local reactions. Thus, a new intravenous (iv) dosage form for $\beta$-E is required in order to reduce these severe adverse effects.

One of the most important objectives of this study was to prevent $\beta$-E-induced injection irritation in order to improve patient compliance. $\beta$-E-NLC suspensions were evaluated in an ear vein test, with injection of Elemene injection and saline solution used as positive and negative controls, respectively. A slight vascular injury was observed at the injection site because of venipuncture trauma in all three groups ${ }^{29}$ Two days after the injection, edema and erythema were macroscopically observed at the injection site of the positive control. However, there was no obvious discoloration or damage at, or away from, the injection site in either the $\beta$-E-NLC groups or the negative control group. Results of the histopathological examination are shown in Figure 4, and indicate no apparent morphological changes in the $\beta$-E-NLCtreated group (Figure 4B) compared to the saline-treated controls (Figure 4A). In contrast, vascular angiectasia and inflammatory cell infiltration were observed in the positive control group (Figure 4C). All of these results indicate that $\beta$-E-NLCs caused lesser venous irritation compared to that caused by the Elemene injection.

It has been reported that venous irritation can be reduced by loading drugs into lipids. ${ }^{30,31}$ The presence of large amounts of free $\beta$-E in Elemene injections results in direct contact of the drug with the vein thereby causing venous toxicities. In the present study, $\beta$-E was incorporated into lipid nanoparticles and direct contact between $\beta$-E and the vascular wall was prevented. Thus, determination of EE was very important in characterizing the $\beta$-E-NLCs. Taken together, the experiments described in this study suggest that NLCs are ideal carriers for $\beta$-E and demonstrate reduced venous irritation.

\section{Pharmacokinetics}

Pharmacokinetic studies were performed to compare the $\beta$-ENLCs to an Elemene injection by determining drug concentration in rat plasma up to 80 minutes after iv administration. The plasma concentration-versus-time curves for the $\beta$-E-NLCs and Elemene injection are shown in Figure 5, which displays a one-compartment model with a weight factor of $1 / \mathrm{cm}^{3}$. As shown in Figure 5, mean plasma concentrations decreased biexponentially in both groups. At the first two time points, mean plasma concentrations in the group treated with $\beta$-E-NLCs was similar to that in the group injected with Elemene injec-
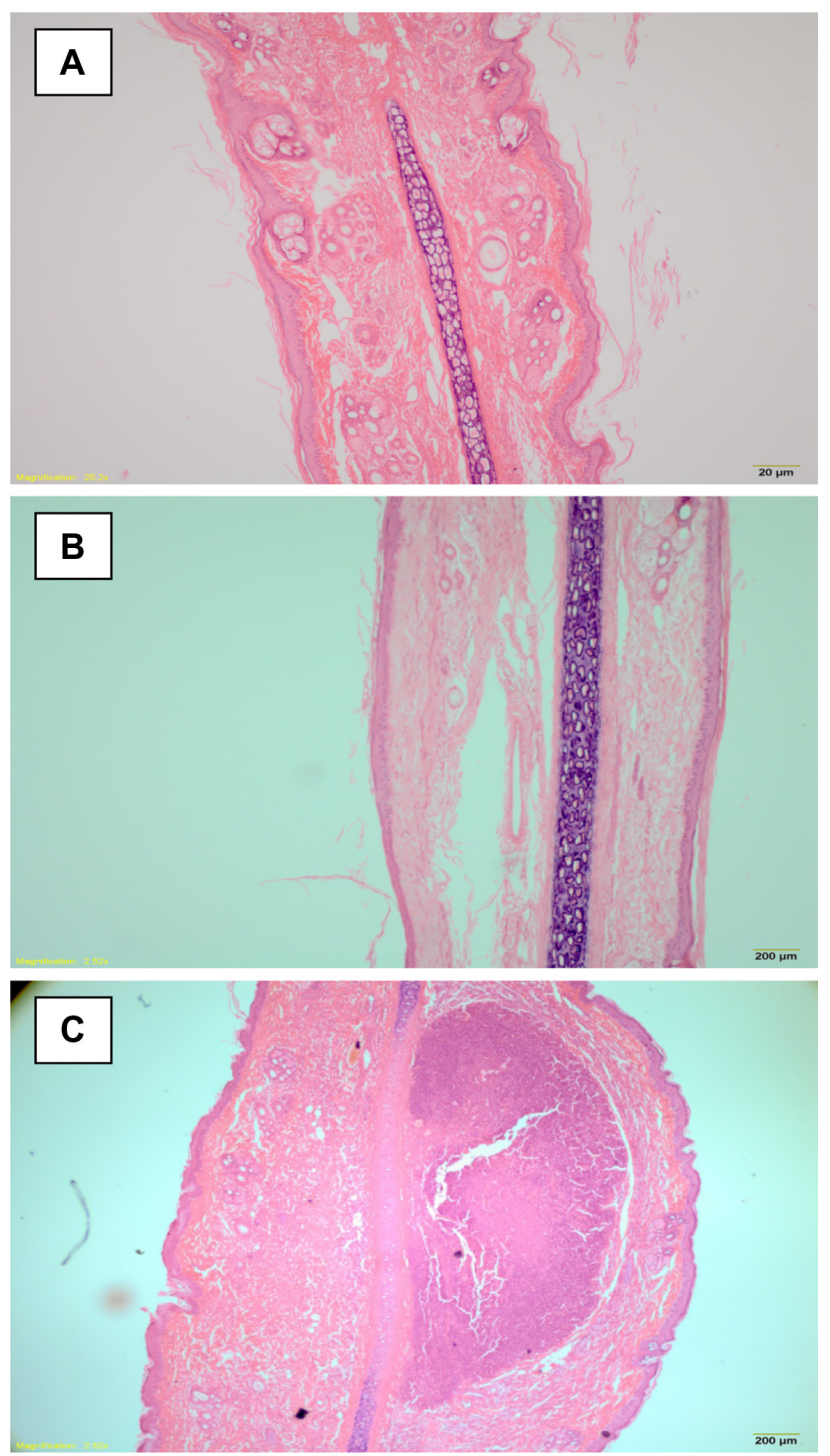

Figure 4 Histopathological slides of the rim of the rabbit ear with the auricular vein following different infusions: normal saline (A), $\beta$-E-NLCs (B), and Elemene injection (C).

Abbreviation: $\beta$-E-NLCs, $\beta$-Elemene-nanostructured lipid carriers.

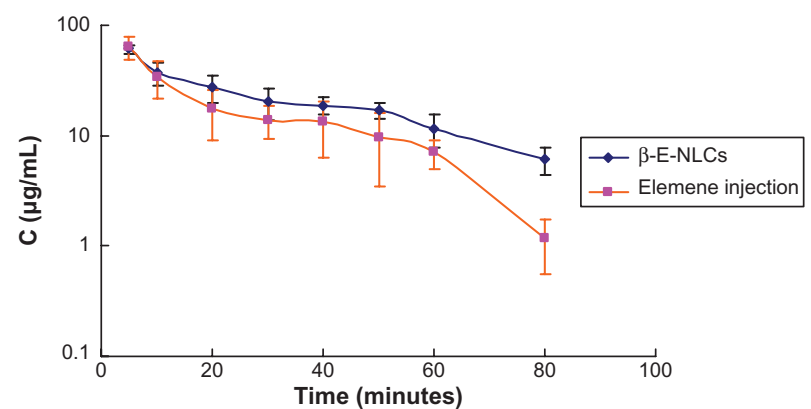

Figure 5 The mean plasma concentration of $\beta$-E-versus-time semilog curves after an intravenous injection of $\beta$-E-NLCs and Elemene injection. Each point value represents the mean and the vertical bars represent the standard deviation $(n=6)$. Abbreviations: $\beta$-E-NLCs, $\beta$-Elemene-nanostructured lipid carriers; $C$, concentration. 
tion. However, at all of the other time points, plasma $\beta-\mathrm{E}$ concentrations were higher in the $\beta$-E-NLC group than in the Elemene injection group.

The main pharmacokinetic parameters examined in this study are summarized in Table 3. Parameters, such as $T_{1 / 2}$, showed no significant differences between the two groups $(P>0.05)$. However, compared to the Elemene injection group, the $\beta$-E-NLC group demonstrated a 1.5-, 1.8-, and 3.5 -fold decrease in $\mathrm{K}_{\mathrm{e}}$ (elimination rate constant), Vss, (steady-state apparent volume of distribution), and CL, respectively. In addition, the AUC after administration of the $\beta$-E-NLCs was 2.5 times higher than after injection of Elemene $(P<0.05)$. Lipid nanoparticles usually exhibit a slow release since the loaded drug is required to transfer from the lipid to an aqueous phase. Thus, $\beta$-E-NLCs produced higher plasma concentrations. Additionally, the $\beta$-E in $\beta$-ENLCs is incorporated into solid and liquid lipids, which could reduce its distribution and tissue penetration. ${ }^{32}$ The higher AUC and slower clearance of $\beta$-E-NLCs compared to the Elemene injection demonstrated that the availability of the $\beta-\mathrm{E}$ is increased by formulation into NLCs.

\section{Anti-tumor efficacy}

Since our pharmacokinetic studies revealed that the availability of $\beta$-E-NLCs was enhanced over Elemene injection, we hypothesized that formulation into NLCs may offer an improved therapeutic index. To confirm this hypothesis, $\mathrm{H}_{22}$ tumor-bearing mice were used to assess whether $\beta$-E-NLCs exhibited higher anti-tumor efficacy. Measurements of tumor weight and inhibition of $\mathrm{H}_{22}$ in mice are shown in Figure 6A. Compared to the saline group, the other three groups showed significant inhibition of the growth of $\mathrm{H}_{22}$-solid tumors, and the tumors were considerably lighter $(P<0.05)$. Furthermore, there was a significant difference in the anti-tumor efficacy of $\beta$-E-NLCs and Elemene injections, with the

Table 3 Pharmacokinetic parameters in rats after intravenous administration of $\beta$-E-NLC suspension and Elemene injection

\begin{tabular}{llrc}
\hline Parameters & Unit & \multicolumn{1}{c}{$\beta$-E-NLCs } & \multicolumn{1}{c}{ Elemene injection } \\
\hline $\mathrm{K}_{\mathrm{e}}^{\mathrm{a}}$ & $\mathrm{L} / \mathrm{hour}$ & $\mathrm{I} .50 \pm 0.2 \mathrm{I}$ & $2.26 \pm 0.40$ \\
$\mathrm{~V}_{\mathrm{ss}}^{\mathrm{a}}$ & $\mathrm{mL}$ & $46.17 \pm 8.13$ & $8 \mathrm{I} .60 \pm 16.73$ \\
$\mathrm{~T}_{\mathrm{I} / 2}$ & minute & $28.82 \pm 3.88$ & $19.53 \pm 4.44$ \\
$\mathrm{AUC}^{\mathrm{a}}$ & $\mu \mathrm{g}$ hour $/ \mathrm{mL}$ & $30.32 \pm 5.74$ & $12.30 \pm 4.33$ \\
$\mathrm{CL}^{\mathrm{a}}$ & $\mathrm{L} /$ hour & $0.1 \mathrm{I} \pm 0.06$ & $0.38 \pm 0.05$ \\
\hline
\end{tabular}

Notes: The data are represented as the mean \pm standard deviation $(n=6)$; $p<0.05$. Abbreviations: AUC, area under the concentration-time curve; $\beta$-E-NLCs, $\beta$-Elemene-nanostructured lipid carriers; $C L$, clearance; $K_{\mathrm{e}}$, elimination rate constant; $\mathrm{T}_{1 / 2}$, half-life; $\mathrm{V}_{\mathrm{ss}}$, steady-state apparent volume of distribution.

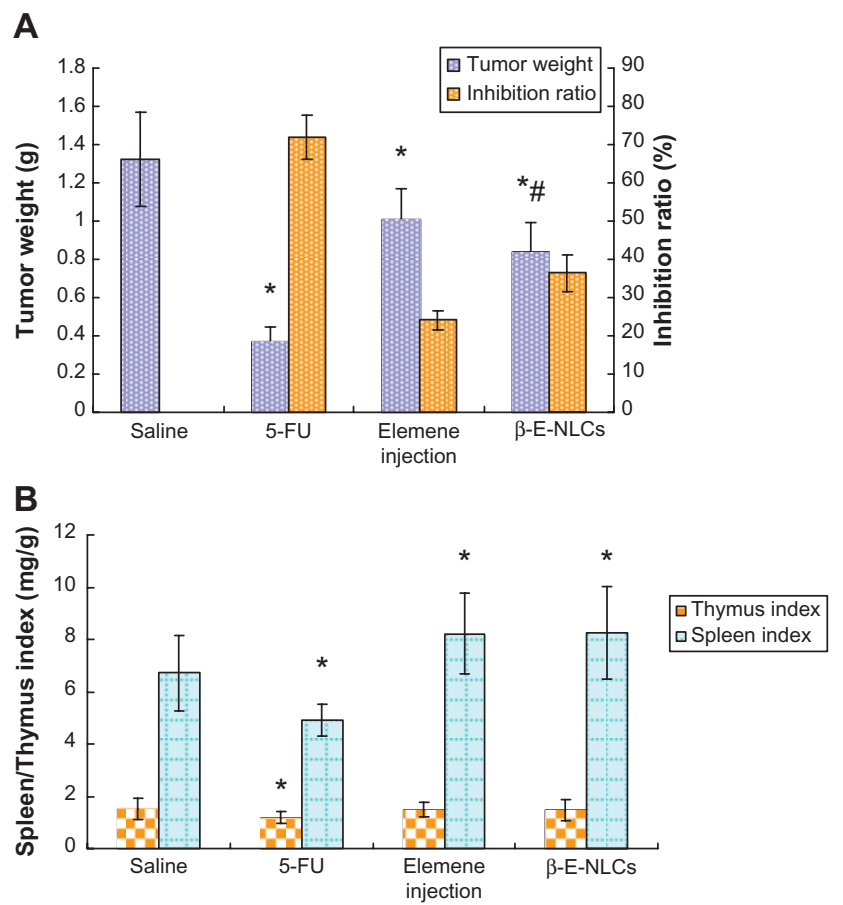

Figure 6 Evaluation of the anti-tumor efficacy $(\mathbf{A})$ and the thymus/spleen indices (B) in tumor-bearing mice.

Notes: $* P<0.05$ compared to the saline group, and ${ }^{\# P}<0.05$ compared to the Elemene injection group.

Abbreviations: $\beta$-E-NLCs, $\beta$-Elemene-nanostructured lipid carriers; 5-FU, Fluorouracil.

resultant inhibition of tumor growth being $34.36 \%$ and $24.11 \%$, respectively $(P<0.05)$. These results suggest that the anti-tumor efficacy of $\beta$-E-NLCs is enhanced, an effect that may be attributed to increased bioavailability.

It is well known that anti-tumor efficacy can be promoted by strengthening the immune system. ${ }^{33}$ It has been previously reported that Chinese herbal medicines can produce antitumor effects by mediating the immune system. ${ }^{34}$ Thus, two of the primary immune organs, ie, the spleen and thymus, were also investigated in this study. As shown in Figure 6B, significant reductions $(P<0.05)$ in both the spleen and thymus indices were observed in the 5-FU group compared to saline controls. Although there was no apparent difference in the thymus index between saline controls and the drugtreated groups (groups treated with $\beta$-E-NLCs and Elemene injection), there was a significant enhancement $(P<0.05)$ of the spleen index in both $\beta$-E-NLCs and Elemene injection treated groups, indicating that $\beta$-E may enhance the immune responses via effects in the spleen.

Previous studies have demonstrated that drug-loaded nanoparticles can improve drug delivery to tumors via passive targeting, which can result in more drugs entering the interior of the cells. ${ }^{35}$ Although future investigative stud- 
ies are required to examine the organ distribution of the $\beta$-E-NLCs, our current data confirm that NLCs represent an ideal delivery system for enhancing the anti-tumor efficacy and reducing venous irritation.

\section{Conclusion}

In this study, $\beta$-E-loaded NLCs with high EE were prepared by high-pressure homogenization. The production technology and formulation were optimized. The $\beta$-E-NLCs produced were spherical with a mean size of approximately $138.9 \mathrm{~nm}$. While administration of $\beta$-E alone was associated with significant problems, including venous irritation and low bioavailability, these problems could be resolved by loading the $\beta$-E into NLCs. Furthermore, the formulated $\beta$-E-NLCs exhibited considerably higher anti-tumor efficacy. Thus, the NLCs developed in this study demonstrate excellent potential as an intravenous delivery system for $\beta$-E.

\section{Acknowledgments}

This work was supported by the Subject Chief Scientist Program (10XD14303900) from the Science and Technology Commission of the Shanghai Municipality, and Program (No IRT1071) for Changjiang Scholars and Innovative Research Team at universities from the State Education Ministry, People's Republic of China.

\section{Disclosure}

The authors report no conflicts of interest in this work.

\section{References}

1. Ajazuddin, Saraf S. Applications of novel drug delivery system for herbal formulations. Fitoterapia. 2010;81(7):680-689.

2. Liu D, Liu Z, Wang L, Zhang C, Zhang N. Nanostructured lipid carriers as novel carrier for parenteral delivery of docetaxel. Colloids Surf B Biointerfaces. 2011;85(2):262-269.

3. Lønning PE. Study of suboptimum treatment response: lessons from breast cancer. Lancet Oncol. 2003;4(3):177-185.

4. Li DC, Zhong XK, Zeng ZP, et al. Application of targeted drug delivery system in Chinese medicine. J Control Release. 2009;138(2):103-112.

5. Tian BC, Zhang WJ, Xu HM, et al. Further investigation of nanostructured lipid carriers as an ocular delivery system: in vivo transcorneal mechanism and in vitro release study. Colloids Surf B Biointerfaces. 2013;102:251-256.

6. Patlolla RR, Chougule M, Patel AR, Jackson T, Tata PN, Singh M. Formulation, characterization and pulmonary deposition of nebulized celecoxib encapsulated nanostructured lipid carriers. J Control Release. 2010;144(2):233-241.

7. Zhang WL, Gu X, Bai H, Yang RH, Dong CD, Liu JP. Nanostructured lipid carriers constituted from high-density lipoprotein components for delivery of a lipophilic cardiovascular drug. Int J Pharm. 2010;391(1-2):313-321.

8. Tiwari R, Pathak K. Nanostructured lipid carrier versus solid lipid nanoparticles of simvastatin: comparative analysis of characteristics, pharmacokinetics and tissue uptake. Int J Pharm. 2011;415(1-2):232-243.
9. Zhang R, Tian A, Zhang H, Zhou Z, Yu H, Chen L. Amelioration of experimental autoimmune encephalomyelitis by ?-elemene treatment is associated with Th17 and Treg cell balance. J Mol Neurosci. 2011;44(1):31-40.

10. Bao F, Qiu J, Zhang H. Potential role of $\beta$-elemene on histone $\mathrm{H} 1$ in the H22 ascites hepatpma cell line. Mol Med Report. 2012;6(1): 185-190.

11. Peng X, Zhao Y, Liang X, et al. Assessing the quality of RCTs on the effect of beta-elemene, one ingredient of a Chinese herb, against malignant tumors. Contemp Clin Trials. 2006;27(1):70-82.

12. Zhao J, Li QQ, Zou B, et al. In vitro combination characterization of the new anticancer plant drug beta-elemene with taxanes against human lung carcinoma. Int J Oncol. 2007;31(2):241-252.

13. Wang G, Li X, Huang F, et al. Antitumor effect of beta-elemene in non-small-cell lung cancer cells is mediated via induction of cell cycle arrest and apoptotic cell death. Cell Mol Life Sci. 2005;62(7-8): 881-893.

14. Chen ZH, Song YX, Che JJ, et al. Validation of a sensitive gas chromatographic-mass spectrometric method for the simultaneous determination of $\beta$-elemene and $\beta$-elemenal in human plasma. J Chromatogr B. 2009;877(4):408-414.

15. Sun YH, Liu GF, Zhang YQ, Zhu H, Ren YF, Shen YM. Synthesis and in vitro anti-proliferative activity of beta-elemene monosubstituted derivatives in HeLa cells mediated through arrest of cell cycle at the G1 phase. Bioorg Med Chem. 2009;17(3):1118-1124.

16. Zhang XY, Deng YJ, Zhao CJ, Wang XM, Li Z, Gao XF. [Study on pharmacokinetics of PVP coated beta-elemene liposome in rats]. Zhong Yao Cai. 2006;29(2):157-160. Chinese.

17. Liu Y, Wang P, Sun C, et al. Wheat germ agglutinin-grafted lipid nanoparticles: preparation and in vitro evaluation of the association with Caco-2 monolayers. Int J Pharm. 2010;397(1-2):155-163.

18. Zhuang CY, Li N, Wang M, et al. Preparation and characterization of vinpocetine loaded nanostructured lipid carriers (NLC) for improved oral bioavailability. Int J Pharm. 2010;394(1-2):179-185.

19. Hu FQ, Jiang SP, Du YZ, Yuan H, Ye YQ, Zeng S. Preparation and characteristics of monostearin nanostructured lipid carriers. Int J Pharm. 2006;314(1):83-89.

20. Li J, Nie S, Yang X, Wang C, Cui S, Pan W. Optimization of tocol emulsions for the intravenous delivery of clarithromycin. Int J Pharm. 2008;356(1-2):282-290.

21. Shi F, Zhao JH, Liu Y, Wang Z, Zhang YT, Feng NP. Preparation and characterization of solid lipid nanoparticles loaded with frankincense and myrrh oil. Int J Nanomedicine. 2012;7:2033-2043.

22. Siekmann B, Westesen K. Melt-homogenized solid lipid nanoparticles stabilized by the nonionic surfactant tyloxapol. I. Preparation and particle size determination. Pharm Pharmacol Lett. 1994;3:194-197.

23. Müller RH, Mäder K, Gohla S. Solid lipid nanoparticles (SLN) for controlled drug delivery - a review of the state of the art. Eur J Pharm Biopharm. 2000;50(1):161-177.

24. Guo CY, Yang CF, Li QL, et al. Development of a Quercetin-loaded nanostructured lipid carrier formulation for topical delivery. Int $J$ Pharm. 2012;430(1-2):292-298

25. Elsie O, Tiwari SB, Udupa N. Niosome entrapped $\beta$-cyclodextrin methotrexate complex as a drug delivery system. Int J Pharm. 1999;31(4):279-284.

26. Teeranachaideekul V, Souto EB, Junyaprasert VB, Müller RH. Cetyl palmitate-based NLC for topical delivery of Coenzyme Q(10) development, physicochemical characterization and in vitro release studies. Eur J Pharm Biopharm. 2007;67(1):141-148.

27. Müller RH. [Zeta potential and particle charge in laboratory practice.] Zetapotential und Partikelladung in der Laborpraxis. Stuttgart: Wissenschaftliche Verlagsgesellschaft; 1996. German.

28. Westesen K, Siekmann B, Koch MHJ. Investigation on the physical state of lipid nanoparticles by synchrotron X-ray diffraction. Int $J$ Pharm. 1993;93(1-3):189-199.

29. Yoh K, Niho S, Goto K, et al. Randomized trial of drip infusion versus bolus injection of vinorelbine for the control of local venous toxicity. Lung Cancer. 2007;55(3):337-341. 
30. Wang Y, Mesfin GM, Rodríguez CA, et al. Venous irritation, pharmacokinetics, and tissue distribution of tirilazad in rats following intravenous administration of a novel supersaturated submicron lipid emulsion. Pharm Res. 1999;16(6):930-938.

31. Su M, Zhao M, Luo Y, et al. Evaluation of the efficacy, toxicity and safety of vinorelbine incorporated in a lipid emulsion. Int J Pharm. 2011;411(1-2):188-196.

32. Zhang HY, Tang X, Li HY, Liu XL. A lipid microsphere vehicle for vinorelbine: Stability, safety and pharmacokinetics. Int J Pharm 2008;348(1-2):70-79.
33. Gao ZW, Zhang DL, Guo CB. Paclitaxel efficacy is increased by parthenolide via nuclear factor-kappaB pathways in in vitro and in vivo human non-small cell lung cancer models. Curr Cancer Drug Targets. 2010;10(7):705-715.

34. Feng L, Jia XB, Shi F, Chen Y. Identification of two polysaccharides from Prunella vulgaris L. and evaluation on their anti-lung adenocarcinoma activity. Molecules. 2010;15(8):5093-5103.

35. Morille M, Montier T, Legras P, et al. Long-circulating DNA lipid nanocapsules as new vector for passive tumor targeting. Biomaterials. 2010;31(2):321-329.
International Journal of Nanomedicine

\section{Publish your work in this journal}

The International Journal of Nanomedicine is an international, peerreviewed journal focusing on the application of nanotechnology in diagnostics, therapeutics, and drug delivery systems throughout the biomedical field. This journal is indexed on PubMed Central,

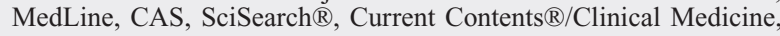

\section{Dovepress}

Journal Citation Reports/Science Edition, EMBase, Scopus and the Elsevier Bibliographic databases. The manuscript management system is completely online and includes a very quick and fair peer-review system, which is all easy to use. Visit http://www.dovepress.com/ testimonials.php to read real quotes from published authors.

Submit your manuscript here: http://www.dovepress.com/international-journal-of-nanomedicine-journal 